\title{
Optical Absorption in Periodic InN:In Structures
}

\author{
D.S. Plotnikov, T.V. Shubina, V.N. Jmerik, A.N. Semenov \\ AND S.V. IVANOV
}

Ioffe Physico-Technical Institute, Russian Academy of Sciences

St. Petersburg 194021, Russia

Optical absorption measurements were exploited to study periodic InN:In structures grown by plasma-assisted molecular beam epitaxy with the thickness of the metallic inclusions varied from 2 to 48 monolayers. We demonstrate that the observed higher-energy shift of an effective absorption edge may be due to In depletion of the InN matrix via the coalescence of In into large clusters, accompanied by the respective higher-energy shift of the Mie resonance. The relevant uncertainty in the optical gap of $\mathrm{InN}$ is discussed.

PACS numbers: 78.66.--w, 71.45.Gm

\section{Introduction}

Currently, InN is considered as a conventional narrow-gap semiconductor with a band width of nearly $0.7 \mathrm{eV}$ [1]. A deviation of $\mathrm{InN}$ band gap value is explained in terms of the Burshtein-Moss effect applied to a non-parabolic band structure [2]. However, a lot of controversial properties of $\mathrm{InN}$ can be explained only in assumption that this material is a metal-semiconductor composite [3]. Indium clusters in an InN matrix are spontaneously formed during growth due to several reasons, such as the small heat of formation, the weak N-In bonds, and difficulties in removing In atoms from the surface. In such small conductive particles one can observe the resonant light absorption - due to so-called Mie resonances, which arise from the interaction of an incident electromagnetic wave with multipolar excitations of electrons in the clusters. The Mie resonances will modify the complex dielectric function of this material and disguise its true adsorption edge $[3,4]$. Besides, specific absorption related to the optical transitions between parallel band in the polyvalent metal [5] has to contribute to the optical losses as well. Such absorption within indium starts from $0.6-0.7 \mathrm{eV}$, i.e. at energy which is very close to the currently accepted fundamental absorption edge of InN. 
This paper is intended to elucidate the role of these effects on optical absorption of InN using specially formed InN:In periodic structures (PSs) with intentionally introduced In inclusions of various thicknesses.

\section{Experimental details}

A set of InN:In PSs were grown by plasma-assisted molecular beam epitaxy using the Compact 21T (Riber) setup equipped by a plasma source HD-25 from Oxford Appl. Res. The growth procedure has been described elsewhere [3]. The substrate temperature was $480^{\circ} \mathrm{C}$. The PSs composed of 20 planar metallic In insertions were equally separated by $25 \mathrm{~nm}$ thick $\mathrm{InN}$ layers. The nominal thickness of the deposited In within the metallic insertions was varied from 2 to 16 monolayers (ML). One sample was composed of 6 insertions of 48 ML. To exclude a spontaneous formation of In clusters these samples were grown in slightly $\mathrm{N}$-riched regimes. However, the tendency to the spontaneous cluster formation allows the precipitation of metallic phase even in this non-balanced condition. A reference sample without In inclusions has been grown and studied for comparison at the same technological parameters.

Optical absorption measurements have been exploited to detect the difference in these two types of structures: the InN epilayer and InN:In. The studies were carried out using a two-monochromators setup SDL-2, comprising a closed-loop helium cryostat (all measurements were taken at 25-30 K). A tungsten lamp was used as a light source, while several detectors (photomultipliers, InGaAs and $\mathrm{PbS}$ photodiodes) were used for registration of the transmitted signal in different spectral ranges. Optical absorption in the structures was characterized by their optical density. For each sample two spectra were measured - one to measure the light transmitted through the sample and another to determine the apparatus function. The latter was done using a set of calibrated neutral filters. Based on preliminary reflectivity measurements, the reflection losses are assumed to be about $20 \%$ of the total intensity.

\section{Results and discussions}

The main result of the absorption measurements is that the light is transmitted through the PSs, even when the total thickness of the metallic insertions amounts to $90 \mathrm{~nm}$. The light attenuation is deficient as compared with the expected one for absorption in a continuous metallic film (absorption coefficient of about $10^{6} \mathrm{~cm}^{-2}$ ). This permits us to conclude that the insertion represents rather an array of flattened clusters than a continuous layer. The modification of morphology takes place presumably during the process of growth.

A set of absorption spectra is presented in Fig. 1 for the PS samples with different thicknesses of the In insertions.

One can see that there is a variation in the absorption between samples which cannot be explained in the framework of previously used theories. We believe that 


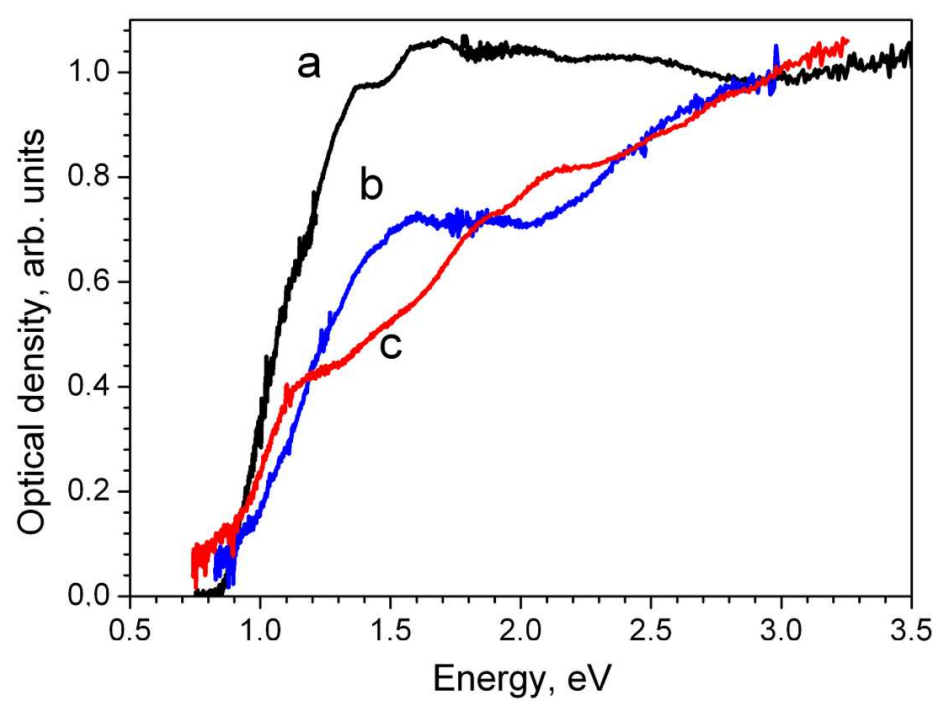

Fig. 1. Selected absorption spectra measured in conventional $\mathrm{InN}$ epilayer $(a)$ and two periodic InN:In PSs: $(b)$ - with 8 ML thick insertions and $(c)$ - with 48 ML thick insertions.

such variation can be realized, when there is the combined effect of the indium deposition, which includes not only the plasmonic effect on the resulting optical properties, but also the effect on the growth process. The deposited indium atoms, from one side, can locally change the composition and, from the other side, can serve as nucleation centers.

For rough consideration of the process we can use the model of the kinetics of precipitation from supersaturated solid solutions proposed by Lifshitz and Slyozov in [6], with supersaturation of the matrix by In characterized by a factor $\Delta$.

The diffusion effect may induce the formation and coalescence of metallic grains. For each value of the supersaturation, there exists a critical radius

$$
R_{\mathrm{C}}=\alpha / \Delta,
$$

which corresponds to equilibrium between the grain and solution. The grains with $R>R_{\mathrm{C}}$ grow, while those with $R<R_{\mathrm{C}}$ dissolve. The constant $\alpha$ depends on the inter-phase surface tension $\sigma$, the temperature $T$, the atomic volume of the solute $\nu$, and the concentration of the saturated solution $C$ :

$$
\alpha=(2 \sigma / k T) \nu C .
$$

Both supersaturation level and critical radius vary in time. The critical radius at the zero time corresponds to the mean size of formed grain without taking the process of coalescence into consideration.

We have estimated the critical radius at the zero time in this simplest model assuming $\Delta \approx 1 \%$ and the constant growth temperature $T=480^{\circ} \mathrm{C}$. It turns out to be of about $10 \mathrm{~nm}$, which is on the order of the size of metallic clusters 


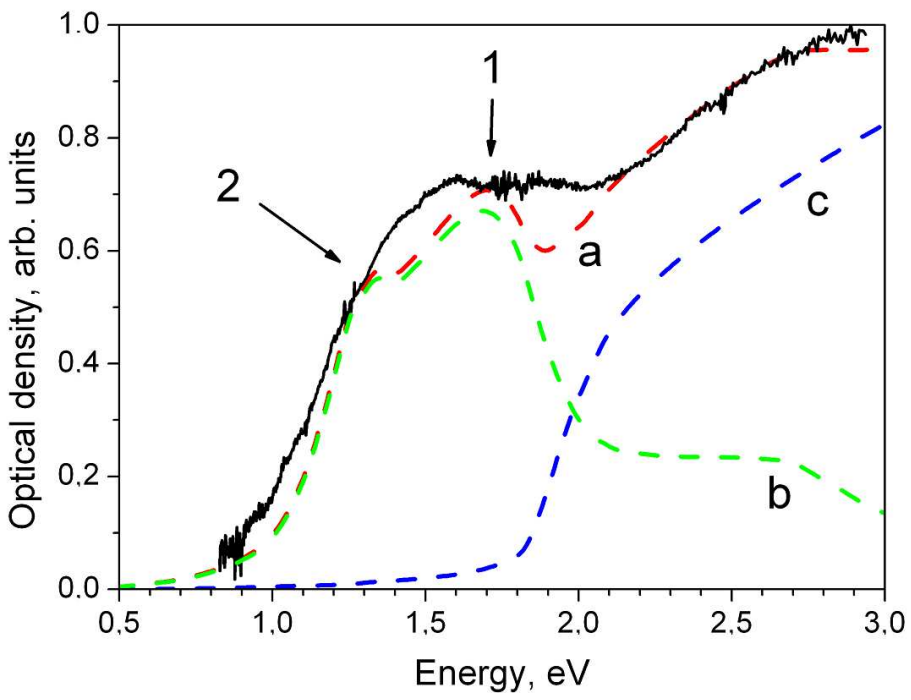

Fig. 2. Optical density of a sample with a nominal insertion thickness of 8 ML. Dashed lines show theoretical fitting. (a) A simulated spectrum; (b) a component of absorption related to the In-clusters; $(c)$ an InN absorption spectrum used in this modeling.

reported previously. They were formed spontaneously on $\mathrm{InN} /$ sapphire interface and visualized by scanning electron microscopy study [7].

In our structure, each $25 \mathrm{~nm}$ layer of $\mathrm{InN}$ is bounded by the planar array of the metallic clusters. We suppose that these clusters can attract the metallic atoms from the environment. Thus, finally, we will observe the arrays of clusters of increased thickness, as compared with the nominal insertion. The adjusting InN layers, in turn, will be depleted by In. In recent papers (see, e.g. [8]) it has been shown that non-stoichiometry of In:N has a strong influence on the observed band gap of the material. Namely, one should expect the higher-energy shift with the $\mathrm{N} / \mathrm{In}>1$ [9]. We believe that this effect provides a general shift of the absorption edge in the InN:In PSs with respect to the reference samples. Because it is difficult to prevent the formation of ultra-small In clusters, characterized by the absorption between the parallel bands, the uncertainty in the $\mathrm{InN}$ optical gap still exists even in the case of conventional epilayers.

We have made an attempt to fit the absorption spectra using the effective medium approximation as it has been done previously [10]. This model can be used when the inclusions are much smaller than the light wavelength. The clusters are approximated by ellipsoids. The expression for the frequency-dependent complex dielectric function of a metal/semiconductor composite with the ellipsoidal clusters was taken from [11]. We assume the filling factor of the metal fraction to be equal to $1 \%$. Due to the current uncertainty and possible non-stoichiometry, the complex dielectric function of $\mathrm{InN}$ is assumed to be an adjusting parameter. The best fitting 
of our spectra corresponds to the optical gap of $1.8 \mathrm{eV}$. This value is close to the former band-gap value of $1.9 \mathrm{eV}$, obtained, likely, on non-stoichiometric samples. The experimental dielectric function of indium determined at $4 \mathrm{~K}$ has been taken from [12].

Figure 2 presents a spectrum of optical density for the sample with $8 \mathrm{ML}$ insertions with its fitting. One can see the background interband absorption in InN along with the contributions related to the In clusters. The latter contains obviously two components: the one (marked "1" in Fig. 2) with the constant peak at $1.5 \mathrm{eV}$ is related to the parallel band absorption within In, while the other (marked "2") is due to the Mie resonance, whose energy is dependent on the depolarization factor, i.e. on the cluster shape. From this fitting we obtained the depolarization factor $L_{\mathrm{m}}$ of mean cluster, it appears to be about 0.14 . Such small $L_{\mathrm{m}}$ can be realized with thin oblate inclusions elongated in the growth plane [13]. It corresponds approximately to the short/long axis ratio of $1 / 5$.

\section{Conclusion}

In conclusions, the investigation of absorption in specially formed $\mathrm{InN}$ :In structures demonstrates that the In insertion is discontinuous, rather an array of cluster than a film. The deposition of the metallic In dramatically changes the growth conditions resulting in the depletion of a surrounding InN area followed by an increase in effective optical gap. The resulting optical spectra are influenced by both optical extinction effects at the metallic clusters and depletion.

\section{References}

[1] P. Carrier, S.-H. Wei, J. Appl. Phys. 97, 033701 (2005).

[2] J. Wu, W. Walukiewicz, W. Shan, K.M. Yu, J.W. Ager,III, E.E. Haller, Hai Lu, William J. Schaff, Phys. Rev. B 66, 201403 (2002).

[3] S.V. Ivanov, T.V. Shubina, V.N. Jmerik, V.A. Vekshin, P.S. Kop'ev, B. Monemar, J. Crystal Growth 269, 1 (2004).

[4] G. Mie, Ann. Phys. (Leipzig) 25, 377 (1908).

[5] W.A. Harrison, Phys. Rev. 147, 467 (1966).

[6] I.M. Lifshitz, V.V. Slyozov, J. Phys. Chem. Solids 19, 35 (1961).

[7] T.V. Shubina, J. Leymarie, V.N. Jmerik, A.A. Toropov, A. Vasson, H. Amano, W.J. Schaff, B. Monemar, S.V. Ivanov, Phys. Status Solidi A 202, 2633 (2005).

[8] K. Scott, A. Butcher, M. Wintrebert-Fouquet, P.P.-T. Chen, K.E. Prince, H. Timmers, S.K. Shrestha, T.V. Shubina, S.V. Ivanov, R. Wuhrer, M.R. Phillips, B. Momemar, Phys. Status Solidi C 2, 2263 (2005).

[9] T.V. Shubina, S.V. Ivanov, V.N. Jmerik, M.M. Glazov, A.P. Kalvarskii, M.G. Tkachman, A. Vasson, J. Leymarie, A. Kavokin, H. Amano, I. Akasaki, K.S.A. Butcher, Q. Guo, B. Monemar, P.S. Kop'ev, Phys. Status Solidi A 202, 377 (2005). 
[10] T.V. Shubina, S.V. Ivanov, V.N. Jmerik, D.D. Solnyshkov, V.A. Vekshin, P.S. Kop'ev, A. Vasson, J. Leymarie, A. Kavokin, H. Amano, K. Shimono, A. Kasic, B. Monemar, Phys. Rev. Lett. 92, 117407 (2004).

[11] R.W. Cohen, G.D. Cody, M.D. Coutts, B. Abeles, Phys. Rev. B 8, 3689 (1973).

[12] A.I. Golovashkin, I.S. Levchenko, G.P. Motulevich, A.A. Shubin, Sov. Phys.-JETP 24, 1093 (1967).

[13] L.D. Landau, E.M. Lifshitz, Electrodynamics of Continuous Media, Pergamon, Oxford 1984, p. 24. 